\title{
Energy Level Based Stable Election Protocol in Wireless Sensor Network
}

\author{
Yogesh Mishra $^{\# 1}$, Ashish Singhadia ${ }^{\# 2}$, Rashmi Pandey ${ }^{\# 3}$ \\ ${ }^{\#}$ Electronics and Communication Engineering Department, \\ Vedica Institute of Technology, Bhopal, India
}

\begin{abstract}
In Wireless Sensor Network, the energy efficiency is the key issue for designing the protocol because sensor nodes have one time battery backup. There are many modern protocols which extend the lifetime of the wireless sensor network by efficiently using battery power of the sensor node. In this paper, we propose a new strategy and protocol Energy Level Bases Stable Election Protocol (ELBSEP) in Wireless Sensor Network. We analyze and compare the performance and results of modern protocols like LEACH, SEP, ESEP, TEEN and TSEP with ELBSEP. The simulation result shows that performance and throughput of our proposed protocol gives the effective and significant energy efficiency as well as more network lifetime compared to other protocols.
\end{abstract}

Keywords- Wireless Sensor Networks, Clustering, Energy Efficiency, Stable Election, Network Lifetime, ELBSEP.

\section{INTRODUCTION}

Wireless sensor networks (WSNs) [1][2] are group of sensor nodes that sense the environment and send the data to the users. Each sensor node in WSN is an electromechanical sensing device. The microelectronic mechanical systems (MEMS) is a modern advanced technology today and MEMS with wireless communication technologies have developed small sized, low-power andlow-cost multifunctional smart sensor nodes in a wireless sensor network (WSN) [9][10]. For monitoring physical and environmental conditions such as temperature, humidity, radiation, sound, vibration, motion, light and pressure the sensor nodes cooperate together to collect environmental information and data.

Earlier, the developments, application and usage of wireless sensor networks were initiated by military such as battlefield surveillance; today the modern sensor [9][10] networks are bidirectional and have self-controlling ability. Modern sensor networks are used in many industrial, commercial and consumer applications, such as industrial process control and monitoring, instrument health monitoring, healthcare applications, traffic control system, home automation and so on. The WSN [1] consists of hundreds to thousands sensor nodes, where each sensor node is connected to several sensor nodes. Each such sensor node has several components: a radio transceiver, an internal antenna or connection to an external antenna, a microcontroller, an electronic circuit for interfacing to the sensors and a power source, typically a battery or an embedded form of energy source. The cost of sensor nodes may vary, according to the type, size, functionality, applications and complexity of the individual sensor nodes. The cost of the multifunctional sensor is usually higher than the normal single functional sensor node. Size and cost limitations on sensor nodes result in corresponding limits on resources such as power backup, memory, computational speed, processing speed, durability, efficiency, accuracy and communications bandwidth. The network layout and topology of the WSNs may differ from a simple star network to an advanced multi-hop wireless mesh and hybrid network. The information propagation technique among the multiple hops of the sensor network may be routing or flooding. To resolve the scalability and expandability issues the cluster based techniques and protocols have been originally proposed for the wire line networks. Now, the cluster based protocols are used in WSNs to minimize the energy consumption [3]. Once WSN is deployed, then the battery recharge or replacement of sensor nodes are not possible practically. Therefore, WSN must operate without human manipulation or involvement so our main focus is to enhance the lifetime of the network in any way and for this purpose many protocols and techniques were introduced and proposed.

\section{BACKGROUND}

In this paper we reviewed, explored and analyzed some modern energy efficient protocols [19]like LEACH, SEP, ESEP, TEEN and TSEP.

\section{A. LEACH (Low Energy Adaptive Clustering Hierarchy)}

LEACH [4] is a proactive and cluster based routing protocol. In $\mathrm{LEACH}$ clustering reduces the energy consumption in sensor nodes. In a wireless sensor network, to distribute the load evenly among all sensor nodes the hundreds and thousands of sensor nodes are dispersed randomly. These sensor nodes continuously sense data, transmit it to their associated cluster heads ( $\mathrm{CHs}$ ) which receive, aggregate and send this data packets to the Base Station (BS) or sink. In $\mathrm{LEACH}$, all the sensor nodes deployed in the environment are homogeneous and each node has limited battery power. To distribute the work load among all nodes and to improve the lifetime of the network clusters are formed. In this network, each sensor node is made to become CHs on their turns [4]. Each node randomly elect itself as a $\mathrm{CH}$ (cluster head) and this process is done in a way that each node becomes a $\mathrm{CH}$ once in the time period of $1 / P$ round. Once a node becomes cluster head $(\mathrm{CH})$ then again the same node will become $\mathrm{CH}$ after all other nodes get a chance to become a $\mathrm{CH}$. The cluster heads (CHs) selection procedure is done on the probabilistic basis [4], each node generates a random number $r$ in the rage of 0 
and 1 , if generated value is less than threshold value computed by the equation given below [4], and then that node becomes a $\mathrm{CH}$.

$$
T_{N}=\left\{\begin{array}{cc}
\frac{P}{1-P\left[\operatorname{r.mod} \frac{1}{P}\right]} & \text { if } n \in G \\
0 & \text { otherwise }
\end{array}\right.
$$

where,

$\mathrm{T}_{\mathrm{N}}=$ Threshold

$P=$ Probability or change of node to become a $\mathrm{CH}$

$r=$ Current round number

$G=$ Set of nodes which are not became a $\mathrm{CH}$ in 1/P round. By using this threshold value, each sensor node will become a $\mathrm{CH}$ in 1/P rounds, thus probability of becoming $\mathrm{CH}$ among remaining nodes must be increased, and however there are fewer nodes that are eligible to become $\mathrm{CH}$.

\section{Advantages of LEACH:}

- The strategy of LEACH [4] protocol is completely distributed, it minimizes energy consumption 4 to 8 times lower in case of multi-hop data packets transmission.

- All the sensor nodes in the network die at about the same time due to even distribution of $\mathrm{CH}$ work in $\mathrm{LEACH}$ protocol.

- The control information from base station is not required for sensor nodesin LEACH [4]protocol.

- LEACH [4][6] minimizes 7 to 8 times low overall energy consumption as compared to direct transmission and minimum transmission energy (MTE) [6]routing protocol.

- Sensor nodes do not require knowledge of global network or identificationin completely distributed wireless sensor network.

\section{Limitation of LEACH:}

- Nodes have different energy level, but $\mathrm{CH}$ is selected unreasonably.

- The performance of LEACH protocol is not ideal for large geographical areas.

\section{B. SEP (Stable Election Protocol)}

SEP [7] protocol is an improvement and enhancement of LEACH [4] protocol which uses clustering based routing strategy based on the node heterogeneity of the sensor node in the networks. In this protocol and technique, some of the sensor nodes have high energy they are referred to as the advanced nodes and the probability of the advanced nodes to become $\mathrm{CHs}$ is more as compared to the normal nodes and the normal nodes have lower energy as compared to the advanced nodes in the network. SEP strategy uses a distributed method to select a $\mathrm{CH}$ in WSNs. It is heterogeneity-aware protocol and $\mathrm{CH}$ selection probabilities of nodes are weighted by initial energy of each node compared to the other nodes in WSN. So basically, SEP protocol is based on two levels of node heterogeneity as normal nodes and advanced nodes.
- Let, mis the fraction of total number of nodes $\mathrm{n}$, which are deployed with $\alpha$ times more energy than the others nodes.

- These powerful nodes are as advanced nodes.

- The remaining $(1-\mathrm{m}) \times \mathrm{n}$ nodes are as normal nodes.

- Probability of normal nodes to become $\mathrm{CHs}$ is calculated as

$$
P_{\text {nor }}=\frac{P_{\text {opt }}}{1+m \cdot \alpha}
$$

- Probability of advanced nodes to become $\mathrm{CHs}$ is calculated as

$$
P_{a d v}=\frac{P_{o p t}}{1+m \cdot \alpha}(1+\alpha)
$$

$\mathrm{P}_{\text {opt }}$ is the optimal probability of each node to become $\mathrm{CH}$ in the network. In SEP [7][8]strategy, selection of $\mathrm{CH}$ is done randomly on probability basis for each node. Sensor nodes continuously sense data and transmit it to their associated $\mathrm{CH}$ and $\mathrm{CH}$ transmit that data it to the sink or base station (BS). This system can be further improved by increasing the value of mor $\mathrm{P}_{a d v}$. Due to advance nodes with two level of node hetero geneity, SEP [7] strategy results in high stable time period, high network lifetime and high throughput.

\section{Advantage of SEP:}

- Any identification or global knowledge of energy of sensor node is not required in SEP [7] technique at each selection round of cluster head.

\section{Limitations of SEP:}

- The cluster head $(\mathrm{CH})$ selection among sensor nodes are not dynamic, which results that nodes that are far away from the powerful nodes will die first.

\section{ESEP (Enhanced Stable Election Protocol)}

ESEP [7][11] is improvement and enhancement of SEP technique. Three type of sensor nodes are considered in ESEP method, as normal, advance and intermediate nodes on the basis of their energy levels. The purpose of ESEP is to build a self-configured WSN which enhances network lifetime and stability period. Each sensor node in a network, continuously sense environment and transmits data to their associated $\mathrm{CH}$, whereas, $\mathrm{CH}$ aggregates data to reduce data redundancy and sends that data to base station. In ESEP, advance nodes are some of total nodes having additional energy as in SEP. Intermediate nodes are some nodes having some extra energy greater than normal nodes but less than advance nodes, and normal nodes are the remaining nodes. In ESEP, CHs are selected on probability based method for each type of node.

\section{Advantage of ESEP:}

- Due to three levels of heterogeneity in ESEP [7][11], the power saving advantage is little enhanced as compared to SEP.

The limitation of ESEP is same as SEP. 


\section{TEEN (Threshold Sensitive Energy Efficient sensor} Network protocol)

TEEN [13] is a reactive network routing protocol which is basically used for time critical applications. In TEEN [13] protocol, nodes continuously sense the medium, but the data packets are transmitted less frequently. In TEEN [13] strategy, data packets are transmitted only when there is any change occurs in the environment. TEEN [13] is basically threshold sensitive protocol which is based on two levels of threshold value, first hard threshold and second soft threshold. In hard threshold mode, the nodes transmit data packets if the sensed data value exceeds the limited range and thus it reduces the number of data packet transmissions or frequent data transmission. In soft threshold mode, the nodes transmit data packets if there is any little variation in the sensed data value. The sensor nodes continuously sense the environment and store the sensed data value for transmission up to the hard threshold limit exceeds. Whenever the sensed data value equals or exceeds the hard threshold value, then sensor nodes transmit their data packets to associated CHs. Next time, data packets are transmitted if there is any difference between the sensed data value and previously saved data value is equals or exceeds the soft threshold value. So, in TEEN [13] routing strategy, energy consumption is reduced as well as great throughput is achieved, network lifetime is increased and stability time period is improved than proactive based protocols.

\section{Advantages of TEEN:}

- TEEN [13] is well suited for the time critical applications.

- In terms of energy consumption and response time TEEN protocol is quite efficient.

- According to the applications and criteria, soft threshold [14] value can be varied.

- Smaller value of soft threshold produces more precise result of the WSN.

\section{Limitations of TEEN:}

- The sensor nodes will never communicate, if the threshold value is not reached. No data will be achieved from the sensor network at all and it will be unknown even if all the sensor nodes die.

- Cluster heads (CHs) will always wait for data from their nodes and keep their transmitter on.

\section{E. TSEP (Threshold-Sensitive Stable Election Protocol)}

TSEP [12] combines the features of ESEP and TEEN protocols. TSEP is also a reactive routing protocol and it has three different levels of energies. Cluster head $(\mathrm{CH})$ selection is done by threshold value, due to three levels of node heterogeneity and being reactive network routing protocol, it produces increased stability period and network lifetime. By comparing TSEP with SEP, LEACH, ESEP and TEEN it is concluded that TSEP protocol performs well in small as well as large geographical networks.

\section{Advantages of TSEP:}

- TSEP [12] combines the best features of ESEP and TEEN protocols.

- The performance of TSEP is better than LEACH, SEP, ESEP and TEEN protocols.

\section{Limitation of TSEP:}

- There is no calculation of energy levels for cluster head $(\mathrm{CH})$ selection, $\mathrm{CH}$ is still probability based in TSEP protocol.

LEACH, SEP, ESEP, TEEN and TSEP protocols still use probability based cluster head $(\mathrm{CH})$ selection. On probability based cluster head selection, low energy nodes may be selected as cluster head and high energy nodes may not be selected as cluster head. LEACH, SEP and ESEP are proactive network routing protocols where nodes continuously transmit data to base station and transmission consumes more energy compared to sensing. SEP and ESEP are node heterogeneity aware protocols which improve network lifetime but the limitation of node heterogeneity is this that throughput is also increased which decrease lifetime of WSN. TEEN and TSEP are reactive network routing protocols where frequent data transmission is limited by threshold value. To improve energy efficiency, and network lifetime, our proposed protocol ELBSEP is observed to be better than these protocols.

\section{PROPOSED PROTOCOL}

In this section we discuss our new proposed protocol ELBSEP (Energy Level Based Stable Election Protocol) which is based on energy level calculation as well as three levels of node heterogeneity and threshold estimation. Cluster head $(\mathrm{CH})$ selection is based on energy level of nodes in our proposed protocol ELBSEP unlike LEACH, SEP, ESEP, TEEN and TSEP as cluster head is selected on probability bases.

Clustering method [17] provides an efficient and effective way to increase the network lifetime of a WSN. The clustering algorithms discussed in literature review basically utilize two techniques, first the selection of cluster head $(\mathrm{CH})$ with more residual energy and second the rotation of cluster heads $(\mathrm{CHs})$ on the probability basis periodically, for equal distribution of energy consumption among sensor nodes in each cluster and enhance the lifetime of the WSN. To forward data packets to the base station, cluster heads cooperate with other cluster heads, on the probability bases the cluster heads is selected and high residual energy node may not be selected as cluster head $(\mathrm{CH})$ and low residual energy node may be selected as cluster head $(\mathrm{CH})$.

To address this problem, we propose an Energy Level Based Stable Election Protocol (ELBSEP) which is based on residual energy level estimation of sensor nodes as well as it combines the best feature TSEP protocol and also provides mechanism for periodical data packet gathering in WSN.

For cluster formation [16] in the WSN, the base station (BS) broadcasts a signal at a fixed energy level. Each node in the 
network computes its approximate distance from base station based on received signal strength. It provides the sensor nodes to estimate the proper power strength level to communicate with base station [16]. Clusters [16][17] are produced by this clustering formula given below.

$$
R_{c i}=\left(1-c \frac{d_{i}-d_{\min }}{d_{\max }-d_{\min }}\right) R_{\max }
$$

$\mathrm{R}_{\mathrm{ci}}=$ the range of radius in the network for cluster formation, $\mathrm{d}_{\max }=$ maximum distance from sensor node to base station, $\mathrm{d}_{\text {min }}=$ minimum distance from sensor node to base station, $\mathrm{d}_{\mathrm{i}}=$ distance from node $\mathrm{i}$ to base station in WSN, $\mathrm{c}=$ weighted factor (value is between 0 to 1 ),

$\mathrm{R}_{\max }=$ maximum competition radius.

The competition radius of the sensor node is estimated by $\mathrm{d}_{\mathrm{i}}$.If $\mathrm{d}_{\mathrm{i}}$ is bigger, then $\mathrm{R}_{\mathrm{ci}}$ will be smaller. The diameter of the cluster in the WSN dominated by node iis

$$
R_{a}=2 R_{c i}
$$

\section{Cluster heads formation of the network:}

After cluster formation based on the distance from the base station, cluster head is selection process is conducted. Before cluster head selection, sensor nodes are categorized according to the energy levels in the network. ELBSEP is a reactive network routing protocol, as we know the transmission of data consumes more energy than sensing so data transmission is done only when a specific threshold limit is exceeded and it has three levels of node heterogeneity. For three levels of node heterogeneity [20], sensor nodes with different energy levels are:

1) Advanced Nodes

2) Intermediate Nodes

3) Normal Nodes

Advance nodes are some of total nodes which contain additional energy (advance nodes have energy greater than all other nodes). Intermediate nodes are some nodes which contain some extra energy greater than normal nodes and less energy than advance nodes, while normal nodes are the remaining nodes. In the energy model of ELBSEP, we consider following:

Energy of normal nodes $=\mathrm{E}_{0}$

Energy for advance nodes $\mathrm{E}_{\mathrm{adv}}=\mathrm{E}_{0}(1+\alpha)$

Energy for intermediate nodes Eint $=\mathrm{E}_{0}(1+\alpha)$

where, $\mu=\frac{\alpha}{2}$

$\alpha=$ factor for advanced nodes which have $\alpha$ times more energy than normal nodes.

The total energy of normal nodes $=n \cdot b(1+\alpha)$

The total energy of advance nodes $=n . E_{0}(1-m-b . n)$

The total energy of intermediate nodes $=n \cdot m \cdot E_{0}(1+\alpha)$

And finally the total Energy of all the nodes $=n \cdot E_{0}(1-m-$ b.n $)+n \cdot m \cdot E_{0}(1+\alpha)+n \cdot b(1+\mu)=n \cdot E_{0}(1+m . \alpha+b . \mu)$

$\mathrm{n}=$ total number of sensor nodes,

$\mathrm{m}=$ proportion of advanced nodes,

$b=$ proportion of intermediate nodes,

Optimal probability of normal nodes to be selected as cluster head $(\mathrm{CH})$ is calculated by this equation:

$$
P_{\text {nor }}=\frac{P_{\text {opt }}}{1+m \cdot \alpha+b \cdot \mu}
$$

Optimal probability for intermediate nodes to be selected as cluster head $(\mathrm{CH})$ is calculated by this equation:

$$
P_{\text {int }}=\frac{P_{o p t}}{1+m \cdot \alpha+b \cdot \mu}(1+\mu)
$$

Optimal probability for advanced nodes to be selected as cluster head $(\mathrm{CH})$ is calculated by this equation:

$$
P_{a d v}=\frac{P_{o p t}}{1+m \cdot \alpha+b \cdot \mu}(1+\alpha)
$$

$\mathrm{P}_{\mathrm{opt}}=$ Optimal Probability.

For cluster head $(\mathrm{CH})$ selection in our proposed protocol ELBSEP, we improved over TSEP method. In ELBSEP, We have taken threshold levels as the parameters for consideration. Each node generates a random number between 0 and 1, if generated value is less than these threshold then that node becomes a cluster head $(\mathrm{CH})$.

The threshold levels for normal nodes are calculated as

$$
=\left\{\begin{array}{cc}
\frac{P_{\text {nor }}}{1-P_{\text {nor }}\left[\operatorname{r.mod} \frac{1}{P_{\text {nor }}}\right]} \times \frac{E_{\text {current }}}{E_{\text {initial }}} & \text { if } P_{\text {nor }} \in G^{\prime} \\
0 & \text { otherwise }
\end{array}\right.
$$

$\mathrm{G}^{\prime}=$ Set of those normal nodes that have not became cluster head in previous round.

$\mathrm{E}_{\text {current }}=$ Residual energy of the node at current time.

$E_{\text {initial }}=$ Residual energy of the node at initial time.

The threshold levels for intermediate nodes are calculated as

$$
T_{\text {int }}=\left\{\begin{array}{cc}
\frac{P_{\text {int }}}{1-P_{\text {int }}\left[\operatorname{r.mod} \frac{1}{P_{\text {int }}}\right]} \times \frac{E_{\text {current }}}{E_{\text {initial }}} & \text { if } P_{\text {int }} \in G^{\prime \prime} \\
0 & \text { otherwise }
\end{array}\right.
$$

G' = Set of those intermediate nodes that have not became cluster head in previous round.

The threshold levels for advanced nodes are calculated as

$$
=\left\{\begin{array}{cc}
\frac{P_{a d v}}{1-P_{a d v}\left[\operatorname{r.mod} \frac{1}{P_{a d v}}\right]} \times \frac{E_{\text {current }}}{E_{\text {initial }}} & \text { if } P_{a d v} \in G^{\prime \prime \prime} \\
0 & \text { otherwise }
\end{array}\right.
$$

G" = Set of those advanced nodes that have not became cluster head in previous round. 
Here is modification and improvement in our proposed protocol ELBSEP by estimating the ratio of energy levels of node at current time to energy of node at initial time.

Total average number of cluster heads per round $=$ $\mathrm{n}(1-\mathrm{m}-\mathrm{b}) \mathrm{P}_{\text {nor }}+$ n.b. $P_{\text {int }}+$ n.m. $P_{\text {adv }}=n \cdot P_{\text {opt }}$

The proposed protocol ELBSEP has better aspect of energy consumption and improved lifetime of the WSN and this improvement is due to node heterogeneity and ratio of current energy to initial energy of the nodes.

\section{Functioning of network:}

In ELBSEP, at the beginning of each round, node by node cluster head $(\mathrm{CH})$ changes take place. At the time of cluster change, the cluster head $(\mathrm{CH})$ transmits the following parameters [18]:

- Report Time: The time period during which each sensor node successfully transmits the reports.

- Attributes: The set of physical parameters about which information data is being sent.

- Hard Threshold: The upper limit of the value for the sensed attribute beyond which the nodes switch their transmitter on and send reports to their cluster head.

- Soft Threshold: The lowest limit of the value below which the nodes switch their transmitters on and transmit data to their cluster head $(\mathrm{CH})$.

All sensor nodes continuously sense their environment continuously. As the parameters value from attributes equals or exceeds hard threshold limit, transmitter is turned on and the data packets are transmitted to their cluster heads (CHs), however this is for the first time when hard threshold condition is taken place [18]. The sensed parameter value is stored by the sensor node is called the "Sensed Value". The next time, sensor nodes transmit data if the sensed value equals or exceeds the upper limit of the hard threshold or if currently sensed value and the previously sensed value equals or exceeds the limit of soft threshold value. So, by estimating hard threshold and soft threshold, the frequent data packet transmissions can be reduced, as the data transmission will only take place when sensed value equals or exceeds the hard threshold [18]. Further data transmissions is taken place by soft threshold, as it minimize transmissions when there is a small changes in value. Some of important functions and features of our proposed protocol ELBSEP are summarized below:

1.ELBSEP is applicable in time critical applications in which data is sent to the user almost instantaneously.

2. Sensor nodes continuously keep on sensing but data transmission is not continuous as data transmission consumes more energy than sensing and processing, so energy consumption is much less than that of other networks.

3 . When the cluster head changes, the threshold value is calculated byratio of current energy to initial energy of the sensor node, so it is a better strategy for cluster head selection in ELBSEP protocol and values of hard threshold, soft threshold, report time and attributes are transmitted, so user can predict the occurrence of sensed values and parameters according to applications.
4. The attributes value can be varied by the user depending on applications and requirement, as attribute values are broadcasted at the time of cluster head changes.

5. As ELBSEP use ratio of current residual energy to initial residual energy so it balances the energy consumption among sensor nodes and enhances the network lifetime.

The limitation of ELBSEP is that if threshold value is not reached, the base station will not receive any information or data from sensor network and even all the sensor nodes of the network become dead, system will be unknown about this limitations. So, ELBSEP is not useful for those types of applications where a sensed data is required frequently and continuously.

\section{Simulation And Performance Evaluation}

We used MATLAB as a simulator for our implementation and performance evaluation of our proposed protocol ELBSEP. Our purpose of estimating simulations is to compare the performance of ELBSEP with SEP, ESEP, LEACH, TEEN and TSEP protocols on the basis of energy consumption, lifetime of the sensor network and throughput.

Performance attributes used in our MATLAB simulations are as follows:

1.The number of alive nodes during each round.

2.The number of dead nodes during each round.

3.The number of packets sent from cluster heads to the base station,(throughput).

For simulation of LEACH, SEP, ESEP, TEEN and TSEP, we have taken some initial parameter values as well as the same parameter values for our proposed protocol ELBSEP.

Table 1: Initial Parameter Settings
\begin{tabular}{|c|c|}
\hline Parameters & Values \\
\hline $\mathrm{E}_{\text {initial }}$ & 0.60 Joule \\
\hline $\mathrm{E}_{\mathrm{current}}$ & 0.55 Joule \\
\hline $\mathrm{P}_{\mathrm{opt}}$ & 0.10 \\
\hline$\alpha$ & 1.30 \\
\hline $\mathrm{n}$ & 200 \\
\hline $\mathrm{m}$ & 0.20 \\
\hline $\mathrm{b}$ & 0.80 \\
\hline $\mathrm{E}_{0}$ & 0.60 Joule \\
\hline
\end{tabular}

We are considering that initially our WSN consists of 200 sensor nodes, all sensor nodes are placed randomly in a region and a base station (BS) is located at the outside of that region.

For MATLAB simulation, we initialized some parameters like $\mathrm{E}_{\text {initial }}$ as 0.60 Joule, $\mathrm{E}_{\text {current }}$ as 0.55 Joule, $\mathrm{P}_{\text {opt }}$ as $0.1, \alpha$ as $1, \mathrm{n}$ as $200, \mathrm{~m}$ as 0.20 , bas 0.80 and $\mathrm{E}_{0}$ as 0.60 Joule.

On the next MATLAB simulation, we changed the parameters setting to different values. Figure 1 plots the graph of nodes dead during each round. In figure 1, LEACH protocol is shown as the green curve, SEP protocol is shown as the red curve, ESEP protocol is shown as the cyan curve, TEEN protocol is shown as the magenta curve, TSEP is shown as blue curve and our proposed protocol ELBSEP is shown as dashed blue curve. As shown in the figure lour 
proposed protocol ELBSEP has better performance as sensor nodes dies later as compared to other protocol.

In figure 2, same colored curves have been used as in figure 1 for LEACH, SEP, ESEP, TEEN, TSEP and ELBSEP. The graph of nodes alive during each round in figure 2 is the complementary of the graph of nodes dead during each round. Again our proposed protocol ELBSEP performs better as compared to other protocol as shown in the graph. The graph plotted for nodes alive during each round of ELBSEP is shown as again dashed blue curve in figure 2 .

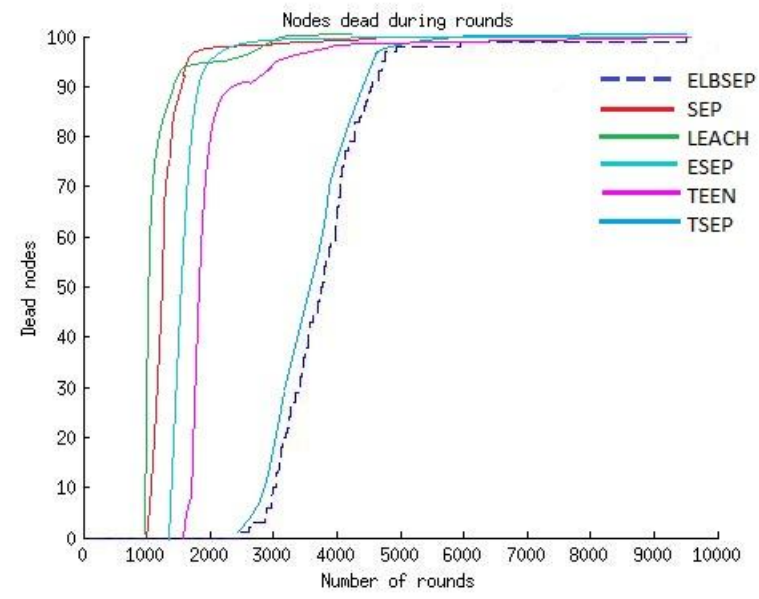

Figure 1: Nodes dead during each round

The graph of figure 3 plots the data packets send to the base station or throughput. Again the same colored curve are used for LEACH, SEP, ESEP, TEEN, TSEP and ELBSEP protocols

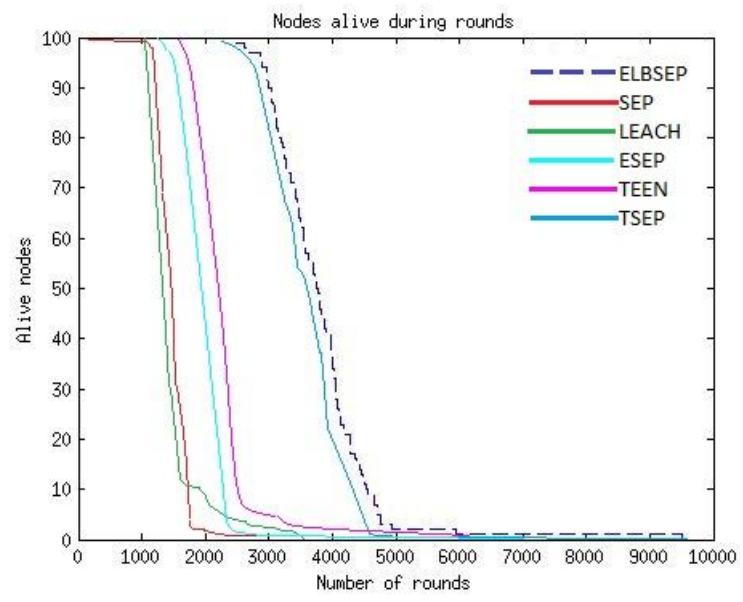

Figure 2: :Nodes alive during each round

For performance evaluation of ELBSEP in MATLAB, we considered the same initial parameter values and the next parameter values as used in LEACH, SEP, ESEP, TEEN and TSEP. As shown in figure 1, the graph plotted for nodes dead during each round in ELBSEP curve shows that our proposed protocol ELBSEP performs better than LEACH, SEP, ESEP, TEEN and TSEP as less nodes die after each rounds as compared to these protocols.

As shown in figure 2, the graph plotted for nodes alive during each round in ELBSEP curve shows that our proposed protocol ELBSEP performs better than LEACH, SEP, ESEP, TEEN and TSEP as more nodes alive after each rounds as compared to these protocols.

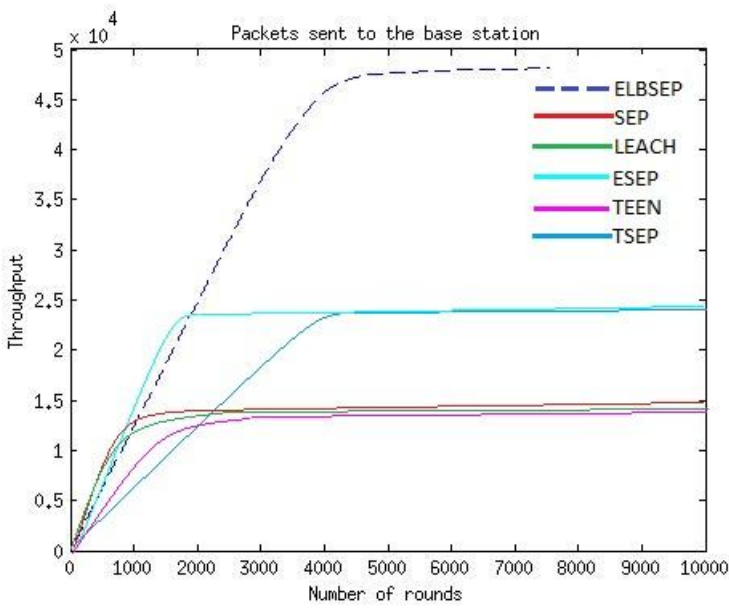

Figure 3: Throughput (Packet sent to base station)

In our MATLAB simulation, we considered the same parameter setting to compare ELBSEP with LEACH, SEP, ESEP, TEEN and TSEP. The throughput of ELBSEP as the graph of data packet sent to the base station is around double as compared to TSEP, as shown in figure 3 which is better than LEACH, SEP, ESEP, TEEN and TSEP. The curve of ELBSEP throughput shows our proposed protocol sends more data packets to the base station (around $50 \%$ more) as compared to other protocols discussed above.

After comparison of ELBSEP with LEACH, SEP, ESEP, TEEN and TSEP, we evaluated that using our proposed protocol ELBSEP, better energy efficiency, enhanced network lifetime and greater throughput.

\section{CONCLUSION ANd Future WORK}

In this paper, we proposed ELBSEP as a reactive network routing protocol with three different levels of node heterogeneity. ELBSEP combines the best features of TSEP and energy level estimation method. Due to the concept of energy level based cluster head selection, hard and soft threshold value, three levels of node heterogeneity and being reactive routing network protocol ELBSEP produces increase in energy efficiency, enhanced lifetime of network and maximum throughput as shown in the simulation result. In comparison with SEP, LEACH, ESEP, TEEN and TSEP it can be concluded that our protocol ELBSEP will perform well in small as well as large geographical networks and best suited for time critical applications.

However ELBSEP is not suitable where frequent information is received from wireless sensor network. Our 
future direction will be to overcome this limitation in this protocol. Finally, in future, the concept and implementation of mobile base station can be introduced in ELBSEP to perform the next level of technology of wireless sensor network.

\section{REFERENCES}

[1] I.F. Akyildiz, W. Su, Y. Sankarasubramaniam, and E. Cayirci, "A Survey on Sensor Network", IEEE Communication Magazine, 2004, pp. 102-114.

[2] Mittal, R.Bhatia, M.P.S., "Wireless sensor networks for monitoring the environmental activities",IEEE International Conference on Computational Intelligence and Computing Research(ICCIC), 2010, pp $1-5$.

[3] Lei Shu, Yan Zhang, Zhangbing Zhou, Manfred Hauswirth, Zhiwen Yu, Gearoid Hynes, "Transmitting and Gathering Streaming Data in Wireless Multimedia Sensor Networks Within Expected Network Lifetime", Mobile Networks and Applications Journal, Volume 13, Issue 3-4, p 323.

[4] Ningbo Wang, HaoZhu, "An Energy Efficient Algorithm Based on LEACH Protocol", International Conference on Computer Science and Electronics Engineering (ICCSEE), 2012, pp. 339-342.

[5] BłażejAdamczyk, "Analysis and Optimization of LEACH Protocol for Wireless Sensor Networks", 20th International Conference on Communications in Computer and Information Science, Vol. 370, pp 86-94.

[6] Ruifeng Zhang,Gorce, Jean-Marie, "Optimal Transmission Range for Minimum Energy Consumption in Wireless Sensor Networks",IEEEConference on Wireless Communications and Networking, WCNC 2008, pp. $757 \quad-762$.

[7] Islam, M M, Matin, M A, Mondol, T K, "Extended Stable Election Protocol (SEP) for three-level hierarchical clustered heterogeneous WSN", IET Conference on Wireless Sensor Systems 2012, pp. 1-4.

[8] O. Rehman, N. Javaid, B. Manzoor, A. Hafeez, A. Iqbal, M. Ishfaq "Energy Consumption Rate based Stable Election Protocol (ECRSEP) for WSNs", Procedia Computer Science, Volume 19, 2013, pp. 932937.

[9] Young, D.J., "Interface electronics for MEMS-based wireless sensing applications", International Symposium on VLSI Design Automation and Test, 2010, pp. 130-133.

[10] Warneke, B.A., Pister, K.S.J., "MEMS for distributed wireless sensor networks", 9th International Conference on Electronics, Circuits and Systems 2002, pp. 291- 294 vol.1.

[11] Raju Pal, RituSindhu, Ajay K Sharma, "SEP-E (RCH): Enhanced Stable Election Protocol Based on Redundant Cluster Head Selection for HWSNs", 9th International Conference on Quality, Reliability, Security and Robustness in Heterogeneous Networks, 2013, Vol. 115, pp. 104-114.

[12] Kashaf, A, Javaid, N., Khan, Z.A, Khan, I.A., "TSEP: ThresholdSensitive Stable Election Protocol for WSNs", IEEE 10th International Conference on Frontiers of Information Technology, 2012, pp. 164 168.

[13] AratiManjeshwar, Agrawal, D.P., "TEEN: a routing protocol for enhanced efficiency in wireless sensor networks", Proceedings 15th International conference on Parallel and Distributed Processing Symposium 2001, pp. 2009 - 2015.

[14] Aliouat, Z, Harous, S., "An efficient clustering protocol increasing wireless sensor networks life time", International Conference on Innovations in Information Technology (IIT), 2012, pp.194 - 199.

[15] Ruihua Zhang, Lei Ju, ZhipingJia, Xin Li, "Energy Efficient Routing Algorithm for WSNs via Unequal Clustering", 14th International Conference on High Performance Computing and Communication \& 2012 IEEE 9th International onference on Embedded Software and Systems, 2012 IEEE, pp. 1226 - 1231.

[16] Chengfa Li, Guihai Chen, Mao Ye, Jie Wu, "An energy-efficien unequal clustering mechanism for wireless sensor networks", IEEE International Conference on Mobile Adhoc and Sensor Systems Conference Improved LEACH Protocol for Wireless Sensor Networks e, 2005, pp. 8-604.

[17] Kumar, N., Kaur, J., "An efficient clustering protocol increasing wireless sensor networks life time”, 7th International Conference on
Wireless Communications, Networking and Mobile Computing, 2011, pp. 1-5.

[18] Baghyalakshmi, D., Ebenezer, J., SatyaMurty, S.A.V., "Low latency and energy efficient routing protocols for wireless sensor networks", International Conference on Wireless Communication and Sensor Computing, 2010, pp. 1-6.

[19] Johnson, M., Healy, M., van de Ven, P., Hayes, M.J., Nelson, J., Newe, T., Lewis, E., "A comparative review of wireless sensor network mote technologies”, IEEE Sensors 2009, pp. 1439-1442.

[20] Gaddam, A., Mukhopadhyay, S.C, Sen Gupta, G, Guesgen, H., "Wireless Sensors Networks based monitoring: Review, challenges and implementation issues", 3rd International Conference on Sensing Technology, 2008, pp. 533-538.

[21] Popovici, E., Magno, M., Marinkovic, S., "Power management techniques for Wireless Sensor Networks: A review", 5th IEEE International Workshop on Advances in Sensors and Interfaces, 2013, pp. $194-198$.

[22] Bin Li, Hongxiang Li, Wenjie Wang, Qinye Yin, Hui Liu, "Performance Analysis and Optimization for Energy-Efficient Cooperative Transmission in Random Wireless Sensor Network", IEEE Transactions on Wireless Communications 2013, Vol. 12, Issue: 9, pp. 4647-4657. 